\title{
Emerging analytical methods to determine gluten markers in processed foods-method development in support of standard setting
}

\author{
Dorcas Weber • Chantal Cléroux • \\ Samuel Benrejeb Godefroy
}

Received: 25 March 2009/Revised: 26 June 2009 / Accepted: 26 June 2009/Published online: 28 July 2009

(C) The Author(s) 2009. This article is published with open access at Springerlink.com

\begin{abstract}
The availability of analytical methods to detect and determine levels of markers of priority allergens in foods is of the utmost importance to support standard setting initiatives, the development of compliance and enforcement activities, as well as to provide guidance to industry on implementation of quality control practices, ensuring the effectiveness of allergen-related sanitation techniques. This paper describes the development and implementation of a mass-spectrometry-based technique to determine markers for individual sources of gluten in beer products. This methodology was shown to answer the requirements of Health Canada's proposed labeling standard for individual gluten source declaration, in order to achieve its policy objectives (i.e., protection of sensitive consumers, while promoting choice). Minimal sample work-up was required and the results obtained by ELISA were further complemented using the LC-MS/MS method. This paper aims to demonstrate the feasibility of alternative techniques to ELISA-based methodologies to determine allergen and gluten markers in food.
\end{abstract}

Keywords Immunoassays · ELISA · Bioanalytical methods $\cdot$ Foods $\cdot$ Beverages $\cdot$ Mass spectrometry

D. Weber $\cdot$ C. Cléroux $\cdot$ S. B. Godefroy $(\bowtie)$

Food Directorate, Health Products and Food Branch,

Health Canada,

251 Sir Frederick Banting Driveway, PA 2203D,

Ottawa, ON K1A 0K9, Canada

e-mail: Samuel.Godefroy@hc-sc.gc.ca

C. Cléroux

e-mail: Chantal.Cleroux@hc-sc.gc.ca

\section{Introduction to celiac disease}

Celiac disease (CD) is an immune mediated disease, triggered in genetically susceptible individuals by the ingestion of gluten. It is also known as celiac sprue or gluten-sensitive enteropathy. Individuals with $\mathrm{CD}$ and dermatitis herpetiformis, a related skin condition, react adversely to the specific proteins of wheat, rye, and barley which are categorized as gluten. Gluten is a generic name given to storage proteins in wheat, barley, rye, and other closely related cereal grains. Prolamins are the aqueous alcohol soluble fraction of gluten isolated from these grains and their hybridized strains (i.e., triticale). In those individuals with $\mathrm{CD}$, these proteins trigger an inflammatory injury in the absorptive surface of the small intestine resulting in malabsorption of protein, fat, carbohydrate, fat-soluble vitamins, folate, and minerals, especially iron and calcium [1-4].

For individuals with $\mathrm{CD}$, exposure to gluten can result in the deterioration, over time, of the cell lining of the small intestine. It is a lifelong condition, and if it is not diagnosed early and treated with a strict gluten-free diet, it can be associated with serious complications, including osteoporosis, lymphoma, and other types of malignancies, infertility in both men and women and a number of autoimmune diseases including insulin dependent diabetes in children. Moreover, in children, $\mathrm{CD}$ can be associated with failure to grow and delayed puberty [2-4]. But beyond the health impacts described above, other impacts such as their effects on quality of life family and social circle are yet to be determined and better understood.

The symptoms and associated conditions of CD vary greatly in number and severity resulting in frequent delays in diagnosis, and misdiagnoses such as irritable bowel syndrome, chronic fatigue syndrome, and fibromyalgia are 
common. A small intestinal biopsy is necessary to confirm the diagnosis. With the advent of new blood tests, the worldwide prevalence of the disease is now estimated to be between $1 / 100$ and $1 / 200$. Certain groups have markedly elevated risks of CD. First-degree relatives of individuals diagnosed with $\mathrm{CD}$ have a $10 \%$ to $20 \%$ risk of developing CD. A high prevalence is also found in those with Down syndrome. Patients with $\mathrm{CD}$ have an increased risk of association with other serious conditions such as Type I diabetes mellitus and other autoimmune disorders $[1,5,6]$.

Although CD is not an IgE-mediated reaction, it fits the general definition of food allergies, which includes all adverse reactions to food, involving an immune mechanism, both IgE-mediated and non-IgE-mediated.

Risk management options for people with celiac disease: avoidance of gluten-containing ingredients, through properly labeled foods

There are currently no known therapeutic options to cure celiac disease, but rather to treat and manage symptoms. The only viable option for celiac disease sufferers is to prevent such symptoms from occurring by avoiding gluten-containing foods. In fact, a strict lifelong avoidance of gluten in the diet is the only effective management of this disease and for the prevention of subsequent complications [1].

In avoiding foods containing the culprit ingredients to which they are likely to react, celiac individuals and wheat allergy suffers must rely on information provided to them by food processors and importers. For prepackaged foods, it is critical that presence of ingredients, specifically those derived from cereals such as wheat, barley, rye, or oats be clearly indicated on the label. Similarly, if the foods are prepared in a manner that makes them suitable for celiac individuals, by excluding wheat, barley, and rye proteins, they could be identified as "Gluten-free" to make them easily identifiable by consumers who seek them. Under these conditions, labeling is identified as a public health tool enabling susceptible consumers to manage avoidance, but also allowing informed choice from safe and nutritious food sources.

\section{Regulatory requirements for labeling of gluten in prepackaged foods available for sale in Canada}

Labeling of prepackaged foods for gluten sources and the identification of "Gluten-free" foods through labeling has been the focus of various labeling regulations and legislations throughout the world, as a tool to help manage celiac disease.
The Codex Committee on Nutrition and Foods for Special Dietary Uses (CCNFSDU) has been engaged in the development of international standards defining gluten free foods. The latest standard was adopted by the Codex Alimentarius Commission at its last meeting in 2008 (CODEX STAN 118-1979-amended in 1983 and revised in 2008) and defines gluten free foods as foods containing less than $20 \mathrm{ppm}$ of proteins derived from wheat, barley, rye and/or any of their hybridized strains.

In Canada, Health Canada has the mandate to establish food standards, policies, regulations, and guidelines. The department has oversight on labeling requirements associated with health and safety or nutritional quality concerns.

The Canadian Food Inspection Agency (CFIA) is responsible for "...the enforcement of the Food and Drugs Act as it relates to food, as defined in section 2 of that Act...", in other words "for...any article manufactured, sold, or represented for use as food or drink for human beings."

A proposed regulatory amendment has recently been developed and published[7], which introduces a regulatory definition for gluten as «any gluten protein from the grain of any of the following cereals or the grain of a hybridized strain created from at least one of the following cereals:»)

- Wheat, spelt, kamut

- Oats

- Barley

- Rye

- Triticale

This regulatory proposal would impose the declaration of any gluten sources (i.e. the cereal) on the food label of processed foods available for sale in Canada, when gluten (as defined by the regulation) is present in the food, as a result of deliberate addition (as an ingredient entering in the composition of the product). This proposal is currently subject to public comments, with its final version planned for implementation in early 2010.

Similarly, Canadian food regulators have been amongst the pioneering regulators to introduce a "Gluten-Free" regulation. Division 24 of the Canadian Food and Drug Regulations (food for specialty dietary purposes) has defined gluten-free products (B24.018). The requirements for a gluten free product according to these regulations are as follows: «No person shall label, package, sell, or advertise a food in a manner likely to create an impression that it is a gluten free food unless the food does not contain wheat, including spelt and kamut, or oats, barley, rye or triticale or any part thereof». This regulation was enacted in 1996 and enabled the availability of gluten-free foods for celiac and wheat allergic individuals. This regulation did not include a threshold for compliance and its enforcement was based on the analytical threshold of the time (i.e. 200 ppm of gluten as detected by enzyme-linked immuno- 
sorbent assay (ELISA)-based methods available during the late 1990s). With the development and availability of more sensitive techniques, this threshold was lowered to $20 \mathrm{ppm}$. This 20-ppm gluten level is based on clinical and serological evidence that chronic exposure of traces of gluten traces in the diet does not cause inflammation of small intestine mucosa or change in small intestinal architecture, and at the same time allowing a margin of safety for the variable gluten sensitivity and dietary habits of the celiac [8]. The gluten-free regulation imposes that manufacturers of gluten-free foods ensure that all ingredients in the gluten free foods comply with the requirements set in the regulations. They must ensure that cross-contamination in the production facility is prevented, particularly when gluten-containing foods are handled. The application of this regulation extends beyond manufacturing conditions to cover merchandising outlets (wholesale and retail), which must also ensure that gluten free products are handled in a manner that enables them to remain gluten-free.

In view of the adoption of the revised gluten free standard by the Codex Alimentarius Commission (CODEX STAN 118-1979-amended in 1983 and revised in 2008), Health Canada is currently reviewing the gluten-free regulations to further align with its proposed regulatory amendments for labeling of allergens, gluten sources, and added sulfites. Current considerations include the use of the same definition for gluten, where the presence of the protein fraction derived from cereal ingredients would be required to trigger the exclusion of the food from bearing the label of "gluten free". Such consideration will allow products to make a gluten-free claim, even though they contain an ingredient deriving from the incriminated cereal, as long as the protein fraction has been removed and is not present. For example, products containing maltodextrin or other sugar-based ingredients deriving from cereals will be able to bear a "Gluten free" claim, therefore enabling more choice. Considerations are also being made for the removal of oats from the list of excluded cereals, while specific requirements are developed for "Gluten-free oats", being defined as free from protein derived from the other cereals such as wheat, rye, or barley [9].

\section{Introduction of new labeling requirements for gluten sources-need for analytical methods}

The availability of analytical methods to detect and determine levels of markers of priority allergens in foods is of the utmost importance to support standard setting initiatives, the development of compliance and enforcement activities, as well as to provide guidance to industry on implementation of quality control practices, ensuring the effectiveness of allergen-related sanitation techniques. In the early 1990s, Health Canada's Food Program launched an ambitious allergen method development program. Methods were developed for the detection of peanut [10, 11], egg [12], hazelnut [13], and crustacean tropomyosin [14]. After the creation of the Canadian Food Inspection Agency (CFIA) in 1997, these methods were transferred to the agency's laboratories and made the backbone of the laboratory capacity in support of the Canadian compliance programs with regards to undeclared allergens in processed foods. Since that time, a number of commercially available allergen test kits have emerged, enabling the determination of markers of priority allergens in a variety of foods. These kits are either based on the immunochemical or PCR approaches, and have become more widely available for an increasing number of different allergens.

While maintaining its capacity for method development and validation, Health Canada's food allergen methodology program has been realigned to support regulation making and standard setting.

As a food regulator, Health Canada's Food Directorate may be challenged about the availability of methodologies to support its proposed regulatory measures and standards associated with labeling of allergen and gluten sources.

In fact, the regulated food industry is to expect from food regulators to be open and transparent about analytical methods, which are meant to support compliance measures. They are also to expect that such methods be available to demonstrate the feasibility of food standards under development and the ability to comply with them. Health Canada was therefore asked by some food stakeholders' guidance on analytical methods that support its proposed labeling standard to declare sources of gluten in food products such as alcoholic beverages and in particular, beer.

\section{Gluten protein in beer products}

Gluten is a class of proteins found in wheat, barley, rye, and other grains. It includes the two groups of proteins known as glutelins and prolamins. Glutelins consist of the lowmolecular-weight glutenins and the high-molecular-weight glutenins with masses ranging from 15 to $150 \mathrm{kDa}$. These proteins contain an abundance of cysteine residues resulting in an increase of intra- and intermolecular disulfide bonding, hence their high molecular weight. When reduced, these glutelins yield subunits of lower molecular masses. The prolamins are monomeric polypeptide chains with molecular weights between 30 to $50 \mathrm{kDa}$. They are rich in proline and glutamine $(20-55 \%)$. Prolamins in wheat are known as gliadins, in barley as hordeins, in oats as avenins, and in rye as secalins. Celiac individuals may also react not only towards gliadins but also towards barley gluten (hordein) and rye gluten (secalin). 
Health Canada's proposed labeling requirements for sources of gluten are meant to enable celiac individuals to identify those foods containing gluten sources, but also to allow those with wheat allergy to clearly distinguish wheatcontaining foods from foods where other gluten sources were used as ingredients. This measure would enable to protect sensitive consumers (both celiacs and individuals with wheat allergy), while promoting their food choices.

Beer products will be subject to Health Canada's proposed measures for declaration of gluten sources. Some stakeholders requested guidance as to the availability of methods to determine markers of gluten in beverages such as beer, given that bioanalytical techniques based on ELISA may not be providing determining results whose purpose is, in particular, to clearly identify the source of gluten. The purpose of this paper is to summarize recent progress accomplished by Health Canada's Food Allergen Research Program in enabling determination and confirmation of gluten protein markers in beverages such as beer products using a combination of protein separation techniques coupled with mass spectrometry.

Since most beers are brewed from barley malt, residual barley proteins, hordein in particular, are expected to be present. Depending on the type of beer however, other gluten protein markers would be more prevalent.

\section{Materials and method}

A method was specifically developed to address the extraction and identification of markers of gluten from beer products.

A minimal sample work-up protocol was used to prepare the sample. An aliquot of degassed beer sample was diluted five to ten times with the extraction solvent ethanol/water (60:40). The solution was vortexed for $30 \mathrm{~s}$ and then centrifuged at $11,000 \mathrm{rpm}$ for $30 \mathrm{~min}$. A $30-\mu \mathrm{L}$ aliquot of the supernatant was digested with trypsin at $37^{\circ} \mathrm{C}$ for $6 \mathrm{~h}$, after reduction with dithiothreitol (DTT) and alkylation with iodoacetamide (IA). The tryptic digest was analyzed by capillary liquid chromatography-tandem mass spectroscopy (LC-MS/MS) using Water's CapLC/QTOF2 system.

\section{Instrumentation}

The LC-MS/MS system used was a Waters CapLC system coupled to a Waters quadrupole-time of flight mass spectrometer (QTOF2) with nanolockspray (Waters, Milford, MA). Detailed experimental parameters were described in our previous work on the mass-spectrometric detection of milk allergens [15].
LC conditions

Tryptic digests of beer samples were analyzed using a combination of a pre-column (OPTI-PAK $0.35 \mathrm{~mm} \times 5 \mathrm{~mm}$ packed with Waters Symmetry 300 C18 $5 \mu \mathrm{m}$ [Waters, Milford, MA]) and an analytical column. Two types of analytical columns were used: traditional 3-5- $\mu \mathrm{m}$ C18 particulate nano columns $(100 \mathrm{~mm} \times 75 \mu \mathrm{m}$ packed with $5 \mu \mathrm{m}$ PepMap C18 100 A [LC Packing/Dionex, Sunnyvale, $\mathrm{CA}])$ and monolithic columns $([0.1 \mathrm{~mm} \times 150 \mathrm{~mm}]$ silica monolithic column Onyx C18 [Phenomenex, Torrance, $\mathrm{CA}]$ ). The LC gradient was carried out with $0.1 \%$ formic acid in acetonitrile:water $(3: 97 ; v: v)$ as solvent $\mathrm{A}$ and $0.1 \%$ formic acid in acetonitrile as solvent B programmed from $5 \% \mathrm{~B}$ to $35 \% \mathrm{~B}$ in $45 \mathrm{~min}$. at approx. $200 \mathrm{~nL} / \mathrm{min}$ flow.

\section{Mass spectrometry conditions}

The mass spectrometer was operated in nanospray ESI+ mode at a resolution of 10,000 with nanolockspray for accurate mass correction (mass error $<5 \mathrm{ppm}$ ). The capillary voltage was set at $3.5 \mathrm{kV}$ and cone voltage at $45 \mathrm{~V}$. The data acquisition was performed using Masslynx 4.0 in survey (DDA - data-directed analysis) mode with MS to MS/MS criteria based on charge state selection $(+2,+3$, and +4$)$.

Data analysis

Three bioinformatic suites were used to analyze the data obtained from the LC-MS/MS process: Proteinlynx Global Server v2.3 (PLGS, Waters, Milford, MA), Mascot (which includes Mascot Distiller, Mascot Deamon, and Mascot Server, a bioinformatics software package from Matrix Science (Matrix Science Inc., Boston, MA)) and Peaks Studio v 4.5 (BSI Bioinformatics Solutions Inc., Waterloo, Ontario). Each package contains its own peak detection software which processes the raw DDA data into de-isotoped peak lists. These precursor ions list with their corresponding MS/MS spectra were then submitted to a database search for protein identification based on the peptides found.

\section{ELISA method}

A commercial ELISA for gluten determination was used to test beer samples and compare results with those generated by mass spectrometry. Test kits were purchased from RBiopharm Inc. MI, USA. The ELISA enabled the determination of gluten protein with a detection level as low as 3 parts per million and with a cross-reactivity to proteins derived from wheat, rye, and barley. Sample extraction and handling followed the manufacturer's instructions 
Table 1 Major proteins identified in the beer digest based on the proteomic approach using mass-spectrometric techniques (criteria for protein detection based on the Mascot score being above the significant hit threshold with three or more peptides matched to the protein)

\begin{tabular}{|c|c|c|}
\hline Sample & Accession & Protein name \\
\hline \multirow[t]{8}{*}{ B08 } & gi|47168353 & Chain A, Non-Specific Lipid Transfer Protein 1 From Barley \\
\hline & gi|108597921 & Dimeric alpha-amylase inhibitor precursor [Triticum aestivum] \\
\hline & gi|89143120 & Putative avenin-like a precursor [Triticum aestivum] \\
\hline & gi|84617213 & Type 2 non-specific lipid transfer protein precursor [Triticum aestivum] \\
\hline & gi|164457873 & High-molecular-weight glutenin subunit y10 [Triticum aestivum $]$ \\
\hline & gi|17425212 & Low-molecular-weight glutenin subunit group 10 type $\mathrm{V}$ [Triticum aestivum] \\
\hline & gi|10638433 & Gamma-gliadin [Triticum turgidum] \\
\hline & gi|7209265 & Alpha-gliadin [Triticum aestivum] \\
\hline \multirow[t]{7}{*}{ B11 } & gi|19039 & LTP $1[$ Hordeum vulgare $]$ \\
\hline & gi| 123970 & Alpha-amylase inhibitor BDAI-1 precursor [Hordeum vulgare] \\
\hline & gi|30421167 & D-Hordein [Hordeum vulgare] \\
\hline & gi|225102 & Trypsin/amylase inhibitor pUP13 [Hordeum vulgare] \\
\hline & gi|288709 & Gamma 3 hordein [Hordeum vulgare] \\
\hline & gi|19079 & Protein Z (180 AA) [Hordeum vulgare] \\
\hline & gi|18929 & B hordein precursor [Hordeum vulgare] \\
\hline \multirow[t]{7}{*}{ B16 } & gi|19039 & LTP 1 [Hordeum vulgare] \\
\hline & gi|585291 & Alpha-amylase/trypsin inhibitor CMd precursor [Hordeum vulgare] \\
\hline & gi|1310677 & Protein z-type serpin [Hordeum vulgare] \\
\hline & gi|226755 & Gamma-hordein [Hordeum vulgare] \\
\hline & gi|30421167 & D-Hordein [Hordeum vulgare] \\
\hline & gi|224385 & hordein B [Hordeum vulgare] \\
\hline & gi|288709 & Gamma 3 hordein [Hordeum vulgare] \\
\hline \multirow[t]{7}{*}{ 2B01 } & gi|47168353 & Chain A, Non-Specific Lipid Transfer Protein 1 From Barley \\
\hline & gi|1310677 & Protein z-type serpin [Hordeum vulgare] \\
\hline & gi|585291 & Alpha-amylase/trypsin inhibitor CMd precursor [Hordeum vulgare] \\
\hline & gi|30421167 & D-Hordein [Hordeum vulgare] \\
\hline & gi|226755 & Gamma-hordein [Hordeum vulgare] \\
\hline & gi|224386 & Hordein B [Hordeum vulgare] \\
\hline & gi|288709 & Gamma 3 hordein [Hordeum vulgare] \\
\hline \multirow[t]{7}{*}{ 2B07 } & gi|123970 & Alpha-amylase inhibitor BDAI-1 precursor [Hordeum vulgare] \\
\hline & gi|19039 & LTP 1 [Hordeum vulgare $]$ \\
\hline & gi|1310677 & Protein z-type serpin [Hordeum vulgare] \\
\hline & gi|30421167 & D-Hordein [Hordeum vulgare] \\
\hline & gi|226755 & Gamma-hordein [Hordeum vulgare] \\
\hline & gi|62484809 & Putative gamma-gliadin [Triticum aestivum] \\
\hline & gi|288709 & Gamma 3 hordein [Hordeum vulgare] \\
\hline \multirow[t]{4}{*}{ B15 } & gi|1310677 & Protein z-type serpin [Hordeum vulgare] \\
\hline & gi|19039 & LTP $1[$ Hordeum vulgare $]$ \\
\hline & gi|226755 & Gamma-hordein [Hordeum vulgare] \\
\hline & gi|75282567 & Serpin-Z7 [Hordeum vulgare] \\
\hline \multirow[t]{7}{*}{ 2B05 } & gi|1310677 & Protein z-type serpin [Hordeum vulgare] \\
\hline & gi|19039 & LTP 1 [Hordeum vulgare $]$ \\
\hline & gi|30421167 & D-Hordein [Hordeum vulgare] \\
\hline & gi|123970 & Alpha-amylase inhibitor BDAI-1 precursor [Hordeum vulgare] \\
\hline & gi|226755 & Gamma-hordein [Hordeum vulgare] \\
\hline & gi|123459 & B3-hordein [Hordeum vulgare] \\
\hline & gi|288709 & Gamma 3 hordein [Hordeum vulgare] \\
\hline B17 & gi|20159 & $19 \mathrm{kDa}$ globulin precursor [Oryza sativa] \\
\hline
\end{tabular}


Table 1 (continued)

\begin{tabular}{lll}
\hline Sample & Accession & Protein name \\
\hline gi|20210 & Glutelin [Oryza sativa] \\
gi|29839419 & $13 \mathrm{~S}$ globulin seed storage protein 3 precursor [Fagopyrum esculentum] \\
gi|83416591 & $16 \mathrm{kDa}$ allergen [Fagopyrum esculentum] \\
\hline
\end{tabular}

\section{Results and discussion}

Eight different beer products purchased from local liquor stores, in Ottawa, Ontario (Canada) were analyzed by LCMS/MS after digestion with trypsin. The data was processed with the bioinformatics suite from Matrix Science. NCBInr, a non-redundant database of protein sequences from the National Center for Biotechnology Information, Bethesda, MD (NCBI) was used in the Mascot MS/MS ion search. All but one beer showed that the most predominant proteins present were from barley. Table 1 shows a partial list of significant hits from the Mascot search. Of all the beers analyzed, gluten from barley (various homologs of hordein including B- and D-hordein and gamma-hordein) were identified in almost all of them. In one of the beers (B08), alpha-gliadin, gamma-gliadin, high-molecularweight (HMW) glutenin and low-molecular-weight glutenin, which constitute wheat gluten, were detected. In addition, other barley proteins such as the lipid transfer proteins (LTP) and protein z (a major albumin of barley grain) were found.

Trace amounts of wheat gluten was also found in one of the beer (2B07) not claimed to be a wheat beer.

One of the beers tested was found to be truly wheat- and barley-free as claimed on the label and contained mainly proteins from buckwheat and rice.

These raw data were also analyzed using the other two bioinformatics suites: PLGS and Peaks Studio. Similar proteins were identified in all three searches hence increasing the confidence in the results. Similar results were obtained using chymotrypsin as the proteolytic enzyme (data not shown).

Results from LC-MS/MS methods were also compared with data obtained using ELISA, which is currently the most common approach for allergen and gluten analysis. The comparison between results obtained from two types of methodologies is summarized in Table 2. As expected, the wheat beer sample gave a high response with the gluten-ELISA. Other samples such as B11, B16, 2B01, and 2B07 also gave a quantifiable response in ELISA suggesting the presence of gluten. However, the LC-MS/ MS enabled to pinpoint the source of such gluten as barley gluten. The cross-reactivity observed with the ELISA method is overcome by the specificity of the MS-based technique. While samples B15 and 2B05 showed a very low response with the ELISA, gluten from barley was formally detected using the LC-MS/MS method. These beer products would correspond to the category of items where the gluten source declaration would be most useful. In fact, these products would be suitable for consumption to wheat allergic consumers (absence of wheat proteins) since $76 \%$ of wheat allergic consumers only react to wheat alone [16], while being unfit for consumption for celiac individuals.

For sample B17, both methods indicated that there is no gluten, but in addition, the LC-MS/MS method identified proteins from rice and buckwheat which agrees with the claim cited on the product label. This confirmation technique would support the suitability of this type of product to both celiac individuals and wheat allergic consumers.

Table 2 ELISA and LC-MS/MS results for detecting gluten in beer

\begin{tabular}{llll}
\hline Sample\# & Beer type/ Country of origin & Gluten-ELISA & LC-MS/MS, major proteins ID \\
\hline B08 & Wheat beer/Canada & High & HMW glutenin (wheat), LTP (wheat), alpha-gliadin (wheat) \\
B11 & Barley beer/Denmark & $74 \mathrm{ppm}$ & D-hordein, gamma 3 hordein, LTP 1 \\
B16 & Barley beer/Canada & $22 \mathrm{ppm}$ & D-hordein, gamma 3 hordein, LTP 1 \\
2B01 & Barley beer/Canada & $6 \mathrm{ppm}$ & D-hordein, gamma 3 hordein, protein z \\
2B07 & Barley beer/Canada & $11 \mathrm{ppm}$ & D-hordein, gamma 3 hordein, LTP 1 \\
B15 & Barley beer/Mexico & Low & Gamma-hordein, protein z \\
2B05 & Barley beer/USA & Low & D-hordein, Gamma-hordein, B3-hordein \\
B17 & Buckwheat beer/Canada & nd & Glutelin (Oryza sativa), major storage protein (buckwheat)
\end{tabular}




\section{Summary and conclusion}

Beer products were analyzed using a proteomics approach based on a mass spectrometry technique to detect and identify sources of gluten. This methodology was shown to answer the requirements of Health Canada's proposed labeling standard for individual gluten source declaration, in order to achieve its policy objectives (i.e., protection of sensitive consumers, while promoting choice). Minimal sample work-up was required and the results obtained by ELISA were further complemented using the LC-MS/MS method.

This paper provides a demonstration of the feasibility of methods to support enforcement and compliance with the proposed standard for labeling of gluten sources. It also shows the feasibility of alternative techniques to ELISAbased methodologies for confirmation purposes. Current efforts are underway to enable the use of similar methods for quantification of gluten protein markers in food.

Open Access This article is distributed under the terms of the Creative Commons Attribution Noncommercial License which permits any noncommercial use, distribution, and reproduction in any medium, provided the original author(s) and source are credited.

\section{References}

1. Zarkadas M, Scott FW, Salminen J, Ham Pong A (1999) Common allergenic foods and their labelling in Canada - a review. Can J Allergy \& Clin Immunol 4(3):118-141

2. Briani C, Samaroo D, Alaedin A (2008) Celiac disease: from gluten to autoimmunity. Autoimmunity Reviews 7:644-650

3. Presutti RJ, Cangemi R, Cassidy HD, Hill DA (2007) Celiac disease. Am Fam Physician 76:1795-1802

4. Kagnoff M (2007) Celiac disease: pathogenesis of a model immunogenetic disease. J Clin Investigation 117(1):41-49
5. Catassi C, Krystal D, Otto Louis J et al (2007) Detection of celiac disease in primary care: a multicenter case-finding study in North America. Am J Gastroenterol 102:1454-1460

6. Frisk G, Hansson T, Dahlbom L, Tuvemo T (2008) A unifying hypothesis on the development of type 1 diabetes and celiac disease: gluten consumption may be a shared causative factor. Med Hypotheses 70:1207-1209

7. Health Canada (2008), Web posting at http://www.hc-sc.gc.ca/fnan/label-etiquet/allergen/guide_ligne_direct_indust-eng.php , "Health Canada urges food manufacturers to label priority food allergens, gluten sources and added sulphites in the prepublication period of the Food Allergen Labelling Regulatory Amendments", last accessed in November 2008

8. Catassi C, Fasano A (2008) Celiac disease. Curr Opin Gastroenterol 24:687-691

9. Health Canada (2007), webposting at http://www.hc-sc.gc.ca/fnan/securit/allerg/cel-coe/oats_cd-avoine-eng.php, "Celiac Disease and the safety of oats", last accessed in November 2008

10. Yeung JM, Collins PG (1996) Enzyme immunoassay for determination of peanut proteins in food products. J AOAC Intern 79(6):1411-1416

11. Newsome WH, Abbott MA (1999) An immunoaffinity column for the determination of peanut protein in chocolate. J AOAC Intern 82(3):666-668

12. Yeung JM, Abbott MA (2000) Determination of egg proteins in food products by enzyme immunoassays. J AOAC Intern 83 (1):139-143

13. Ben Rejeb S et al (2003) Immunochemical-based method for detection of hazelnut proteins in processed foods. J AOAC Intern 86(3):557-563

14. Ben Rejeb S et al (2002) Presentation at the 116th AOAC International Annual meeting and Exposition, Los Angeles, CA, USA, September 22-26, 2002, Abstract \# C-124 p102

15. Weber D, Raymond P, Ben-Rejeb S, Lau B (2006) Development of a liquid chromatography-tandem mass spectrometry method using capillary liquid chromatography and nanoelectrospray ionization-quadrupole time-of-flight hybrid mass spectrometer for the detection of milk allergens. J Agric Food Chem 54 (5):1604-1610

16. Jones SM, Magnolfi CF, Cooke SK, Sampson HA (1995) Immunologic cross-reactivity among cereal grains and grasses in children with food hypersensitivity. J Allergy Clin Immunol 96:341-351 